\title{
CASE REPORT: RETROPERITONEAL FIBROSIS PRECEDED BY NONSPECIFIC CHRONIC PLEURITIS
}

\author{
Elizandra Tomazela Laurenti Polito ${ }^{1, \star}$, Luiz Fellipe Favoreto Genelhu ${ }^{1}$, Thays Zanon Casagrande ${ }^{1}$, Leticia Resende Brandao ${ }^{1}$ \\ 1.Hospital Estadual Dório Silva, Serra (ES), Brazil. \\ *Corresponding author: eliz.polito@gmail.com
}

\section{BACKGROUND}

We report a rare case of primary retroperitoneal fibrosis (PRF) preceded by nonspecific chronic pleuritis in an elderly man.

\section{CASE REPORT}

A 71-year-old male, brown, diabetic, hypertensive. In 2017, he had chest pain, pleural thickening with bilateral pleural effusion was detected. Pleural biopsy in 2019 revealed moderate inflammatory infiltrate, predominantly lymphocytic, with histiocytes and plasma cells amidst fibrosis. Immunohistochemistry revealed CD20 and CD3 on B and T lymphocytes, respectively. Negative for fungi and tuberculin skin test. No neoplastic infiltration. Findings consistent with nonspecific chronic pleuritis. Opted for expectant management for thoracic surgery. In the follow-up, he presented an anal fistula with indication for surgical correction. Preoperatively, he presented abdominal pain and abdominal CT detected a retroperitoneal mass involving the aorta and iliac arteries. Submitted to laparotomy in January 2021 with several foci of fibrosis, biopsies revealed a multinodular lymphocytic infiltrate represented by $B$ and T cells without atypia. Immunohistochemistry revealed CD20, CD3 on B and T lymphocytes, EBV and IgG4 negative. Findings consistent with PRF. Colonoscopy with edematous proctitis, rectal biopsy showing lamina propria with edema, congestion and lymphocytic infiltrate. He began follow-up in rheumatology with abdominal distension, lower abdominal discomfort, significant edema of the lower limbs and anal fistula. Because he is diabetic, we chose to prescribe azathioprine and prednisone. Patient chose to start only azathioprine. Initial tests: 27.1\% polyclonal hypergammaglobulinemia, gamma globulins 2.33, PCR 9.1, ANA negative, anti-Ro negative, ANCA negative, FR negative, Hep B, C, HIV, VDRL negative serology, normal IgG4 assay. After two months, he presented significant improvement in lower limb edema and anal fistula closure, negative CRP, chest CT maintaining bilateral pleural effusion.

\section{CONCLUSION}

This case illustrates a nonspecific chronic pleuritis association with PRF, both of which may be part of the same spectrum of fibroproliferative disease, with similar histology of lymphocytic infiltrate in the midst of fibrosis. One possibility for this picture would be IgG4-related disease, ruled out by negative immunohistochemistry and normal serum IgG4 dosage. The idiopathic type is responsible for more than $70 \%$ of the cases of PRF. There are some case reports of PRF and chronic pleuritis, but we did not find an association of PRF with anal fistula in the literature. The good response to the immunosuppressant, with improvement in iliac compression (reduced edema in the legs) and anal fistula closure suggests that it is an inflammatory disease. The follow-up time was too short to assess the response of nonspecific chronic pleuritis.

\section{KEYWORDS}

Retroperitoneal fibrosis, Chronic nonspecific pleuritis, Fibrosis, IgG4-related disease. 